\title{
The Rumen Bacterial Community in Dairy Cows Is Correlated to Production Traits During Freshening Period
}

\author{
Shuai Huang ${ }^{1}$, Shoukun $\mathrm{Ji}^{2}$, Garret Suen ${ }^{3}$, Feiran Wang ${ }^{1}$ and Shengli $\mathrm{Li}^{1 *}$ \\ 'State Key Laboratory of Animal Nutrition, Beijing Engineering Technology Research Center of Raw Milk Quality and Safety \\ Control, College of Animal Science and Technology, China Agricultural University, Beijing, China, ${ }^{2}$ College of Animal Science \\ and Technology, Hebei Agricultural University, Baoding, China, ${ }^{3}$ Department of Bacteriology, University \\ of Wisconsin-Madison, Madison, WI, United States
}

OPEN ACCESS

Edited by:

Xudong Sun,

Heilongjiang Bayi Agricultural

University, China

Reviewed by:

Min Wang,

Chinese Academy of Sciences, China

Xiliang Du,

Jilin University, China

${ }^{*}$ Correspondence:

Shengli $\mathrm{Li}$

lisheng0677@163.com

Specialty section:

This article was submitted to Systems Microbiology,

a section of the journal

Frontiers in Microbiology

Received: 18 November 2020

Accepted: 11 February 2021

Published: 04 March 2021

Citation:

Huang S, Ji S, Suen G, Wang F and Li S (2021) The Rumen Bacterial

Community in Dairy Cows is

Correlated to Production Traits During

Freshening Period.

Front. Microbiol. 12:630605

doi: 10.3389/fmicb.2021.630605
The rumen microbiome plays a vital role in providing nutrition to the host animal, thereby influencing ruminant production. Despite its importance, it is not fully understood how variation in the ruminal bacteria community composition influences dry matter intake (DMI), milk yield and ruminal fermentative parameters in dairy cows, especially during freshening period. Here, we hypothesized that during early lactation, high DMI cows having a different ruminal microbiota than low DMI cows, and that this difference persists over time. To test this, we enrolled 65 fresh and determinzed their DMI using an autofeed intake recording system. Fourteen days after calving, the 10 animals with the lowest $(\mathrm{LFI})$ and the 10 animals with the highest (HFI)-average DMI were selected for further analysis. Rumen fluid was collected from these two cohorts at 1 (Fresh1d) and 14 days (Fresh14d) after calving and their ruminal microbiota were assessed using 16S rRNA sequencing. Volatile fatty acid (VFA) concentrations were also quantified. Comparison of the ruminal microbiotas between Fresh1d and Fresh14d showed that Fresh14d cows had a significantly higher relative abundance of VFA-producing microbes $(P<0.05)$, such as Prevotella_7 and Succinivibrionaceae_UCG-001. This was commensurate with the concentrations of acetate, propionate, butyrate, valerate and total VFAs, were also significantly $(P<0.05)$ increased in Fresh14d cows. We also found that the differences in the ruminal microbiota between LFI and HFI cows was limited, but DMI significantly altered $(P<0.05)$ the relative proportion of bacteria in the families Coriobacteriaceae, and Succinivibrionaceae. Furthermore, specific operational taxonomic units belonging to the Anaeroplasma was significantly $(P<0.05)$ correlated with $\mathrm{DMl}$ and milk yield. Taking together, our findings provide a framework for future studies of freshening period cow that seek to better understand the role of the ruminal microbiota during this critical period in the lactation cycle.

Keywords: ruminal bacteria, fresh cows, dry matter intake, production traits, 16S rRNA sequencing

Abbreviations: ADF, Acid detergent fiber; AP, acetate-to-propionate ratio; DDGS, Dried distillers grains with soluble; DM, Dry matter; DMI, Dry matter intake; Fresh1d, 1 day after calving of fresh cows; Fresh14d, 14 days after calving of fresh cows; HFI, high average feed intake from the fresh cows; LFI, low average feed intake from the fresh cows; NDF, Neutral detergent fiber; NMDS, Non-Metric Multidimensional Scaling; OTUs, Operational Taxonomic Units; TVFAs, total of volatile fatty acids. 


\section{INTRODUCTION}

Dairy cows are important global contributors to agriculture as sources of milk and milk products. A critical stage in the dairy cow production lifecycle is the transition period, which occurs between lactation cycles and spans from 3 weeks before to 3 weeks after calving. During this period, cows undergo dramatic changes in host physiology and nutrient metabolism, which can result in health disorders, reduced dry matter intake (DMI), and lower milk yield. Previous works has documented the influence of diet on host metabolism and physiology during the transition period, but far less is known regarding the impact of the ruminal microbiome, which is a known driver of host production (Weimer, 2015). Importantly, ruminal microbes ferment plant polysaccharides into VFAs, including acetate, propionate, and butyrate, which serve as the major energy source for the cow (Reynolds et al., 1988; Flint et al., 2008). Recent studies reported the difference of rumen microbiota under the different feed intake of lactating dairy cows (Li et al., 2020) and yaks (Shi et al., 2020). However, there are no studies focused on the difference of rumen microbiota between low and high feed intake in dairy cows during the freshening period.

Recently, studies have shown that the rumen microbiota undergoes dramatic and distinct shifts from gestation to lactation. Lima et al. (2015) described these shifts in 115 Holstein dairy cows (67 multiparous and 48 primiparous) from 1 week before postpartum to 1 week after postpartum. Dynamic changes in the structure of the metabolically active rumen bacterial communities were found over the transition period (parturition \pm 3 weeks), likely in response to the dramatic changes in physiology and nutritional factors like DMI and feed composition (Zhu et al., 2017). These findings were supported by another study of 10 primiparous Holstein dairy cows during the transition period, which also found distinct changes in the rumen bacterial composition in response to dietary changes (Zhu et al., 2018). In contrast, a study by Pitta et al. (2014) showed no difference in the dominant ruminal bacterial phyla, families and genera in both primiparous and multiparous cows 1-3 d post-calving and 4 weeks into lactation.

Given this paucity of data, it is clear that more work is required to better understand the influence of the ruminal microbiota during the transition period. In particular, a deeper understanding of the freshening period (2 weeks after parturition) is necessary, as $\sim 50 \%$ of all cows experience low DMI during this period, resulting in a state of negative energy balance (Ferguson, 2001). Increasing evidence showed that improving DMI of fresh cows can alleviate the negative energy balance and increased the downstream milk production of dairy cows (Roche et al., 2013).

Here, we hypothesized that the rumen microbiota of low DMI cows is significantly different from high DMI cows, and that this difference persists over time. To address this, we conducted a study to explore the dynamics of the ruminal microbial community during the freshening period in dairy cows. Specifically, we compared the ruminal microbiota of low DMI fresh cows to high DMI fresh cows in order to identify potential relationships between the ruminal bacteria and DMI.
Understanding the differences between these groups will provide a framework for fresh cows and thereby achieve improved lactation efficiency while reducing the risk for the adverse outcomes that usually persist in low DMI fresh cows.

\section{MATERIALS AND METHODS}

\section{Animals Care and Management}

Sixty-five fresh $(2.40 \pm 0.50$ parity, body condition score $3.58 \pm 0.12$, body weight $612.13 \pm 11.40 \mathrm{~kg}$ ) Holstein dairy cows were selected after calving from a commercial dairy farm herd (Beijing, China). All cows were cohoused and kept in a free stall barn. No drugs or antibiotics were used 3 months prior to the study. All cows had ad libitum access to fresh water and were fed three times daily (07:30, 14:30, and 19:00) with a total mixed ration, as shown in Table $\mathbf{1}$.

\section{Daily Milk Yield and Dry Matter Intake Data Collection}

Individual feed intake was measured by a roughage intake control system (Insentec B.V., Marknesse, Netherlands). Cows were milked thrice daily $(07: 00,14: 00$, and 22:00) by farm staff. Milk

TABLE 1 | Composition and chemical components of the diet used in this study.

\begin{tabular}{|c|c|c|}
\hline Items $^{1}$ & Pre-partum & Fresh \\
\hline \multicolumn{3}{|l|}{ Ingredients, kg } \\
\hline Oat grass & 4.83 & 1.20 \\
\hline Alfalfa hay & - & 3.00 \\
\hline Whole corn silage & 8.62 & 11.20 \\
\hline Flaked corn & 0.87 & 2.00 \\
\hline Corn pellets & 0.87 & 1.18 \\
\hline Soybean meal & 1.84 & 2.97 \\
\hline Soybean hull & 0.50 & 2.00 \\
\hline Sprayed corn husk & 1.40 & 0.18 \\
\hline DDGS & 1.20 & 1.50 \\
\hline $5 \%$ premix & 0.75 & 0.65 \\
\hline Cottonseed & - & 0.50 \\
\hline $5 \%$ anion premix & 0.13 & - \\
\hline Yeast culture XP & 0.15 & 0.20 \\
\hline Molasses & - & 0.50 \\
\hline \multicolumn{3}{|l|}{ Contents, $\%$} \\
\hline Dry matter as fed & 53.39 & 49.32 \\
\hline Crude protein & 14.23 & 16.56 \\
\hline Crude fat & 2.04 & 3.40 \\
\hline ADF & 20.37 & 20.58 \\
\hline NDF & 40.84 & 34.63 \\
\hline $\mathrm{NEL}^{2}(\mathrm{MCal} / \mathrm{kg})$ & 1.58 & 1.72 \\
\hline $\mathrm{Ca}$ & 0.80 & 0.70 \\
\hline$P$ & 0.35 & 0.33 \\
\hline
\end{tabular}

${ }^{1}$ DDGS dried distillers grains with solubles, DM dry matter, NDF neutral detergent fiber, ADF acid detergent fiber, NFC non-fiber carbohydrates, Ca calcium, $P$ phosphorus.

${ }^{2} N E_{L}$ : Nutrients contents was measured value and $N E_{L}$ was calculated value with model from NRC (2001). 
yield was recorded by a milking machine $(2 \times 48$, BouMatic Company, Madison, WI, United States) for each cow.

\section{Grouping and Sampling Period}

All cows were transferred to a new barn from 10 days before calving to allow the animals to acclimate to their new surroundings. The experimental period was from $1 \mathrm{~d}$ to $14 \mathrm{~d}$ after calving. During this period, the 10 cows with the lowest average DMI (LFI) and the 10 cows with highest average DMI (HFI) were selected from the 65 fresh cows. We note that a previous study found that characterizing the ruminal microbiota of 16 cows maintained on the same diet is sufficient to determine meaningful differences within their microbial communities (Jami and Mizrahi, 2012). Therefore, our selection of 20 disparate DMI cows from a cohort of 65 early lactation cows is likely sufficient to detect differences inthe their ruminal microbiotas as it relates to host phenotype. Once lactation began, the LFI and HFI cows were sampled at 1 (Fresh1d, $n=20)$ and 14 (Fresh14d, $n=20$ ) days after calving.

\section{Blood Samples Collection and Measurement}

Blood samples were collected from each cow via tail vein before morning feeding. Samples were centrifuged at 3,000 $\times \mathrm{g}$ for $10 \mathrm{~min}$ to obtain serum and stored at $-20^{\circ} \mathrm{C}$ untile subsequent analysis of glucose, non-esterified fatty acid (NEFA), and $\beta$-hydroxybutyrate (BHBA). Serum samples were analyzed for NEFA and BHBA using a colorimetric kit (Nanjing Jiancheng, Jiangsu, China), and glucose by a GF-D200 automatic biochemical analyzer (Caihong, Shandong, China).

\section{Rumen Fluid Collection and Processing}

Rumen fluid samples were collected from each cow using an oral gastric tube (Ancitech, Winnipeg, MB, Canada) prior to morning feeding (07:00). The sampling device was cleaned thoroughly with fresh warm water after each sampling to avoid cow-to-cow contamination and the first $200 \mathrm{~mL}$ of collected rumen fluid was discarded to avoid saliva contamination. Subsequent rumen fluid was collected and filtered through four layers of cheesecloth. Samples were placed into sterile $50 \mathrm{~mL}$ plastic tubes on wet ice and immediately transported back to the farm office and frozen at $-80^{\circ} \mathrm{C}$ until DNA extraction was performed.

An additional $30 \mathrm{~mL}$ of rumen fluid was transferred into a centrifuge tube and stored at $-20^{\circ} \mathrm{C}$ until VFA analysis. VFA determination was conducted as follows. Rumen fluid was centrifuged at $8,000 \times \mathrm{g}$ at $4^{\circ} \mathrm{C}$ for $15 \mathrm{~min}$ to obtain the supernatant, which was then quantified using gas chromatography as described by Erwin et al. (1961).

\section{Genomic DNA Extraction, Amplification, and Sequencing}

Total genomic DNA was extracted from $1 \mathrm{~mL}$ rumen fluid samples using an OMEGA DNA kit (Omega Bio-Tek, Norcross, GA, United States) according to the manufacturer's specifications. The quality of DNA was confirmed by
$1 \%$ agarose gel electrophoresis. The amplicon library preparation was performed by PCR amplification of the V3-V4 region of the $16 \mathrm{~S}$ rRNA gene using the primers 338F (5'-ACTCCTACGGGAGGCAGCAG- $\left.3^{\prime}\right)$ and 806R (5'-GGACTACNNGGGTATCTAAT-3') including NEBNext adapters sequences, indices and Taq DNA Polymerase as well as AMPure XP Beads (New England Biolabs Inc., Ipswich, MA, United States) (Ren et al., 2017). PCR conditions are as follows: 5 min of denaturation at $95^{\circ} \mathrm{C}$, followed by 28 cycles of $45 \mathrm{~s}$ for denaturation at $95^{\circ} \mathrm{C}, 50 \mathrm{~s}$ for annealing at $55^{\circ} \mathrm{C}$ and $45 \mathrm{~s}$ for elongation at $72^{\circ} \mathrm{C}$ with a final extension at $72^{\circ} \mathrm{C}$ for $10 \mathrm{~min}$. PCRs were performed in triplicate $25 \mu \mathrm{L}$ mixture containing 12.5 $\mu$ L KAPA 2G Robust Hot Start Ready Mix (Kapa Biosystems, Wilmington, MA, United States), $1 \mu \mathrm{L}$ of each primer $(5 \mu \mathrm{M}), 5$

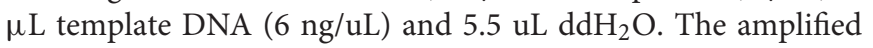
PCR products were purified using an Agencourt AMPure XP Kit (Beckman Coulter Genomics, Indianapolis, IN, United States), and quantified using PCR (ABI 9700, Thermo Fisher Scientific, Waltham, MA, United States). Purified PCR products were pooled in equimolar amounts and sequenced on an Illumina MiSeq (Illumina, San Diego, CA, United States) (Caporaso et al., 2012) using a $2 \times 250$ bp sequencing kit.

\section{Quality Control and Sequencing Data Analysis}

Low quality (score $\leq 20)$ short reads $(<200 \mathrm{bp})$ and reads containing ambiguous bases or unmatched to primer sequences and barcode tags were filtered out from dataset using QIIME 1.8 (Caporaso et al., 2010). The resulting reads were merged using PEAR 0.9.6 (Zhang et al., 2014) and demultiplexed using FLASH 1.20 (Magoc and Salzberg, 2011). Reads with merged length less than $230 \mathrm{bp}$ and chimeric sequences were removed by UCHIME (UCHIME Algorithm) (Edgar et al., 2011). In order to reduce the error caused by the different sequencing depths of the samples, all samples were subsampled to equal size of 23,902 sequences for downstream alpha and beta diversity analysis. To ensure the comparability of the species diversity between the samples, standardized OTU documents were used to analyze the species and diversity indexes.

The remaining sequences were clustered into operational taxonomic units (OTUs) at a 97\% similarity using the Ribosomal Database Project classifier (Cole et al., 2009) with a confidence threshold of 0.70 and compared against the SILVA 128 database (Release September 29, 2016) (Quast et al., 2013). All were removed using UCLUST (Edgar, 2010) to generate a representative OTU table.

The OTU level alpha diversity of bacterial communities was determined using Shannon and Chaol indices and calculated using procedures within QIIME 1.8 and visualized using the "ggplot2" package in R (version 3.6.1) (Wickham, 2009). The non-metric multidimensional scaling (NMDS) ordination was performed on Bray-Curtis dissimilarity distances calculated in R. Analysis of similarities (ANOSIM) (999 permutations) using Bray-Curtis distances were performed to compare the similarity of microbial community among the observed microbial profiles 
based on different groups and sample time using the "vegan" package in R (Oksanen et al., 2015).

\section{Sequence and Statistics Analysis}

Data on DMI, milk yield, rumen fermentation parameters and serum biochemical parameters were analyzed using the linear mixed models procedure of SAS 9.4 (Cary, North Carolina, United States). Alpha-diversity indices, the significance of the pairwise comparison between LFI and HFI groups and between Fresh1d and Fresh14d groups were analyzed using the Wilcoxon rank test using the "dplyr" package $e^{1}$ (author, H. Wickham, R. François, L. Henry, K. Müller; published date, 2018; version, 0.7.6) in R. Spearman's rank correlation was used to identify the relationship between the relative abundance of OTUs and production traits of LFI and HFI cows using the "Psych" package ${ }^{2}$ (author, W Revelle; published date, 2016; version, 1.6.9) and visualized using the "corrplot" package $e^{3}$ (author, Taiyun Wei; published date, 2017; version, 0.84 ) in R. All $P$-value was corrected using a false discovery rate of 0.05 as described by Benjamini and Hochberg (1995) and false discovery rate corrected $P<0.05$ were considered significant.

\section{RESULTS}

\section{Measurement of Production Traits, Rumen Fermentative Parameters, and Blood Metabolites in Fresh Cows}

DMI and milk yield for all cows across the entire trial period are shown in Table 1. The levels of acetate, propionate, butyrate, valerate and total VFAs were significantly $(P<0.05)$ higher in Fresh14d compared to Fresh1d, whereas the acetate:propionate (AP) ratio and serum glucose was significantly $(P<0.05)$ lower in Fresh14d groups. We found that DMI increased and significantly differed $(P<0.05)$ between LFI and HFI groups (Table 2$)$. No significant differences $(P>0.05)$ in milk yield, DMI/milk yield, acetate, propionate, butyrate, valerate, isovalerate, total VFAs, AP, NEFA, BHBA and glucose were observed between LFI and HFI groups.

\section{Sequencing Metrics for the Ruminal Microbiota of Fresh Cows}

A total of $1,087,457$ raw sequences were generated with an average of $27,186 \pm 745$ (mean $\pm S D$ ) per sample, respectively. An average of 1,532 \pm 258 OTUs across all samples was identified at $97 \%$ sequence similarity. Rarefaction curves showed a smaller number of new OTU identification as the number of sequences per sample increased (Supplementary Figure S1), implying the adequate sampling depth for covering the rumen bacterial composition that we tested. Good's coverage for the fresh cow samples was determined with a mean value of 0.982 across all 40 samples, indicating sufficient sequence coverage for all samples.

\footnotetext{
${ }^{1}$ https://cran.r-project.org/package/dplyr

${ }^{2}$ http://cran.r-project.org/web/packages/psych

${ }^{3}$ https://cran.r-project.org/web/packages/corrplot/
}

The mean Shannon's diversity and Chaol's richness for all fresh cow samples was $8.38 \pm 0.64$ and $1963.94 \pm 285.88$, respectively.

The most highly abundant phyla for all fresh cow samples included the Bacteroidetes (52.60\%), Firmicutes (34.90\%), Proteobacteria (6.21\%), with less contributions from the Fibrobacteres (1.24\%) and Spirochaetes $(1.23 \%)$ (Figures $\mathbf{1 A}, \mathbf{B})$. Within these phyla, the most abundant families included the Prevotellaceae (40.17\%), Lachnospiraceae (12.19\%), Ruminococcaceae (9.63\%) and Succinivibrionaceae (5.39\%, Figures 1A,B). At the genus level, 8 genera had $>2 \%$ relative abundance: Prevotella_1 (31.29\%), Succiniclasticum (4.37\%), Christensenellaceae_R7_group (2.78\%), Rikenellaceae_RC9_gut_group (2.69\%), Prevotella_7 (2.53\%), Succinivibrionaceae_UCG-001 (2.22\%), Succinivibrionaceae_UCG-002 (2.04\%), and Ruminococcus_1 $(2.03 \%$, Figures $\mathbf{1 A}, \mathbf{B})$.

\section{Defining a Core Microbiota for Fresh Cows and DMI Cows}

We then sought to determine the core microbiota across all fresh cows in our study and found 2,737 OTUs shared among all fresh cow samples (Figure 2A). These included bacterial families with $>1 \%$ total relative abundance: Prevotellaceae (26.39\%), Lachnospiraceae (6.23\%), Ruminococcaceae (4.26\%), Acidaminococcaceae (4.19\%), Bacteroidales_S24-7_group (3.01\%), Veillonellaceae (2.61\%), Christensenellaceae (1.85\%), and Fibrobacteraceae (1.05\%, Supplementary Table S1). The shared genera among all samples $>1 \%$ of the total relative abundance were the Prevotella_1 (23.39\%), Succiniclasticum (4.19\%), unclassified_Bacteroidales_S24-7_group (3.01\%), Christensenellaceae_R-7_group (1.85\%), Ruminocroccaceae_ NK4A214_group (1.27\%), Lachnospiraceae_NK3A20_group (1.11\%), Selenomonas_1 (1.06\%), and Fibrobacter (1.05\%).

We then determined the core set of OTUs shared between the LFI and HFI cows and found 2,392 OTUs shared across LFI and HFI samples (Figure 2B). More unique OTUs were found in the HFI group, relative to the LFI group. The LFI and HFI groups shared $80 \%$ of the total number of identified OTUs. Of the 2,392 OTUs shared across both DMI groups, most of them belonged to the genera Prevotella_1 (33.26\%), unclassified_c_WCHB1$41(21.80 \%)$, Prevotella_7 (4.96\%), Succinivibrionaceae_UCG001 (4.45\%), unclassified_Bacteroidales_S24-7_group (4.20\%), Succiniclasticum (4.05\%), unclassified_Lachnospiraceae (2.69\%), and Ruminococcus_1 (2.32\%, Supplementary Table S1).

\section{The Ruminal Bacterial Community in Fresh Cows Differs Between Days 1 and 14 After Calving}

To determine if differences exist between the ruminal microbiota of fresh cows at Fresh1d and Fresh14d, we performed a BrayCurtis dissimilarity analysis and visualized this using an NMDS plot as shown in Figure 3A. We found that the ruminal microbiota differed between both groups upon visual inspection. We then analyzed these data in greater detail using ANOSIM and confirmed that these two groups were statistically different $\left(R^{2}=0.65, P=0.001\right)$. Moreover, we found that the ruminal 
TABLE 2 | DMI, milk yield, rumen fermentative parameters and blood metabolites.

\begin{tabular}{|c|c|c|c|c|c|c|c|c|}
\hline \multirow[t]{2}{*}{ Item $^{1}$} & \multicolumn{2}{|c|}{ Freshening period } & \multirow[t]{2}{*}{ SEM } & \multirow[t]{2}{*}{$P$-value } & \multicolumn{2}{|c|}{ DMI } & \multirow[t]{2}{*}{ SEM } & \multirow[t]{2}{*}{$P$-value } \\
\hline & $\begin{array}{l}\text { Fresh1d } \\
(n=20)\end{array}$ & $\begin{array}{l}\text { Fresh14d } \\
(n=20)\end{array}$ & & & $\begin{array}{c}\text { LFI } \\
(n=10)\end{array}$ & $\begin{array}{c}\text { HFI } \\
(n=10)\end{array}$ & & \\
\hline DMl, kg & 6.43 & 15.36 & 0.60 & $<0.001$ & 16.52 & 19.57 & 0.59 & 0.023 \\
\hline Milk yield, kg & 20.47 & 36.41 & 1.45 & $<0.001$ & 34.64 & 36.07 & 1.00 & 0.382 \\
\hline DMI/milk yield & 0.74 & 0.50 & 0.05 & $<0.001$ & 0.53 & 0.61 & 0.03 & 0.586 \\
\hline \multicolumn{9}{|l|}{ Fermentation } \\
\hline Acetate, mmol/L & 45.18 & 61.31 & 4.18 & 0.015 & 61.50 & 61.13 & 2.96 & 0.668 \\
\hline Propionate, mmol/L & 10.96 & 20.22 & 1.63 & 0.002 & 20.74 & 19.64 & 1.75 & 0.781 \\
\hline Butyrate, mmol/L & 5.77 & 10.25 & 0.75 & $<0.001$ & 10.34 & 10.14 & 0.64 & 0.884 \\
\hline Valerate, $\mathrm{mmol} / \mathrm{L}$ & 0.53 & 1.01 & 0.06 & $<0.001$ & 1.10 & 0.90 & 0.08 & 0.225 \\
\hline Isovalerate, mmol/L & 2.10 & 2.05 & 0.12 & 0.779 & 2.18 & 1.92 & 0.12 & 0.269 \\
\hline TVFAs, mmol/L & 63.14 & 94.20 & 6.89 & 0.005 & 93.86 & 94.74 & 4.95 & 0.936 \\
\hline $\mathrm{AP}$ & 5.32 & 3.26 & 0.32 & $<0.001$ & 3.24 & 3.29 & 0.16 & 0.883 \\
\hline \multicolumn{9}{|l|}{ Metabolites } \\
\hline $\mathrm{NEFA}, \mathrm{mmol} / \mathrm{L}$ & 0.20 & 0.21 & 0.002 & 0.058 & 0.21 & 0.20 & 0.005 & 0.170 \\
\hline $\mathrm{BHBA}, \mathrm{mmol} / \mathrm{L}$ & 1.26 & 1.28 & 0.03 & 0.602 & 1.30 & 1.27 & 0.04 & 0.765 \\
\hline Glucose, mmol/L & 3.65 & 3.11 & 0.12 & 0.025 & 3.14 & 3.08 & 0.12 & 0.815 \\
\hline
\end{tabular}

${ }^{1}$ TVFAs, total of VFAs; AP, the acetate-to-propionate raito; NEFA, non-esterified fatty acid; BHBA, $\beta$-hydroxybutyrate.

microbiota of the Fresh14d cows had a significantly $(P<0.001)$ lower number of OTUs, relative to Fresh1d cows, which was further supported by significant differences in the Chaol richness and Shannon diversity index $(P<0.001)$ values for the two groups (Supplementary Figure S2A).

At the phylum level, the relative abundance of phyla Bacteroidetes, Firmicutes and Proteobacteria showed no significant $(P>0.05)$ difference between Fresh1d and Fresh14d. In contrast, the phyla Actinobacteria and Tenericutes were significantly $(P<0.05)$ decreased (Table 3). At the family level, the predominant family Ruminococcaceae, Bacteroidales_BS11_gut_group, Christensenellaceae and Rikenellaceae were significantly $(P<0.05)$ decreased from Fresh1d to Fresh14 (Table 3 ). The relative abundance of the families Prevotellaceae and Veillonellaceae were significantly $(P<0.05)$ higher in Fresh14d compared to Fresh1d (Table 3). At the genus level, the relative abundance of some genera changed more than 10-fold, including Prevotella_7 (increasing 83.33-fold, $P<0.001$ ), Erysipelotrichaceae_UCG-002 (increasing 3827.75-fold, $P=0.001$ ), and Succinivibrionaceae_UCG-001 (increasing 42,380.95-fold, $P<0.001$, Table 4).

\section{Differences in the Ruminal Bacterial Community Between LFI and HFI Cows Is Limited}

Considering the importance of DMI for the fresh group, we compared LFI and HFI cows from the fresh cow group at $14 \mathrm{~d}$ to determine if differences exist between these cows. First, we performed a Bray-Curtis dissimilarity analysis of the microbiota for LFI and HFI cows and visualized this using an NMDS plot as shown in Figure 3B. We found that the ruminal microbiota was similar as both groups did not show a clear separation, and this was also confirmed using ANOSIM analysis $\left(R^{2}=0.006, P=0.355\right)$. In addition, we found that the ruminal microbiota from those two groups had no significant differences in the Shannon diversity index $(P=0.529)$, Chaol richness $(P=0.684)$, and number of OTUs $(P=0.481)$ for the two groups (Supplementary Figure S2B).

We then quantified the difference between the community composition of the LFI and HFI groups using the Wilcoxon test on the relative abundances for all samples at the phylum and family level. We found that phyla Proteobacteria was significantly $(P=0.043)$ enriched in the LFI group relative to the HFI group (Table 3). At the family level, Coriobacteriaceae (phylum Actinobacteria), and Succinivibrionaceae (phylum Proteobacteria $)$ were significantly $(P<0.05)$ different between groups (Table 3). A higher relative abundance of Erysipelotrichaceae_UCG-002 in the LFI group, relative to the HFI group (Table 4). The relative abundance of Christensenellaceae_R-7_group and Ruminococcaceae_UCG010 were tended to higher $(0.05<P<0.1)$ and the relative abundance of Lachnospira was tended to lower $(0.05<P<0.1)$ in the HFI group relative to the LFI group (Table 4).

\section{Correlation of Ruminal Bacteria With Production and Rumen Fermentative Parameters in LFI and HFI Cows}

To explore the potential roles of ruminal bacteria on production and fermentation, we analyzed the relationship between DMI, milk yield, DMI/milk yield, VFAs (acetate, propionate, butyrate, valerate, isovalerate, total VFAs and AP) and the relative abundance of OTUs using Spearman's rank correlations. All OTUs with relative abundances $<0.01 \%$ of all samples were removed from this analysis. The relationship between OTUs and production and fermentation traits were visualized in a heatmap, 
A

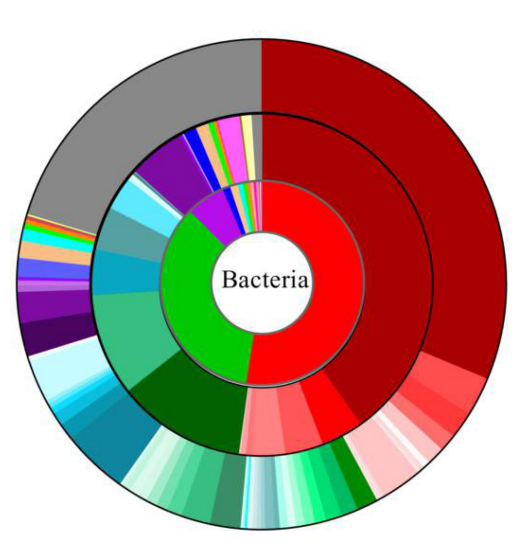

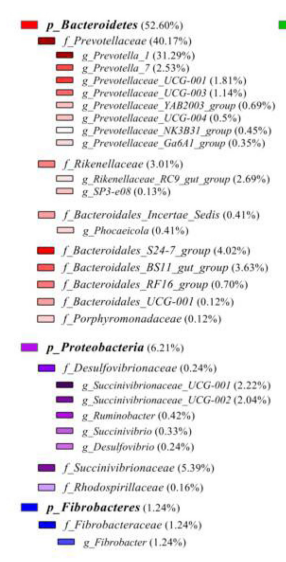

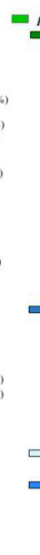

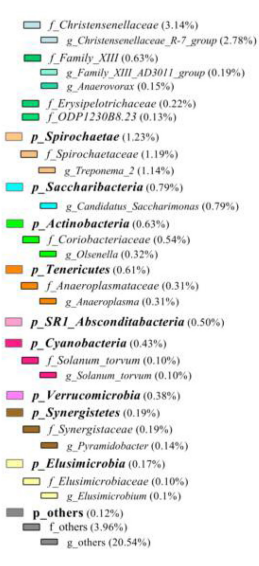

B
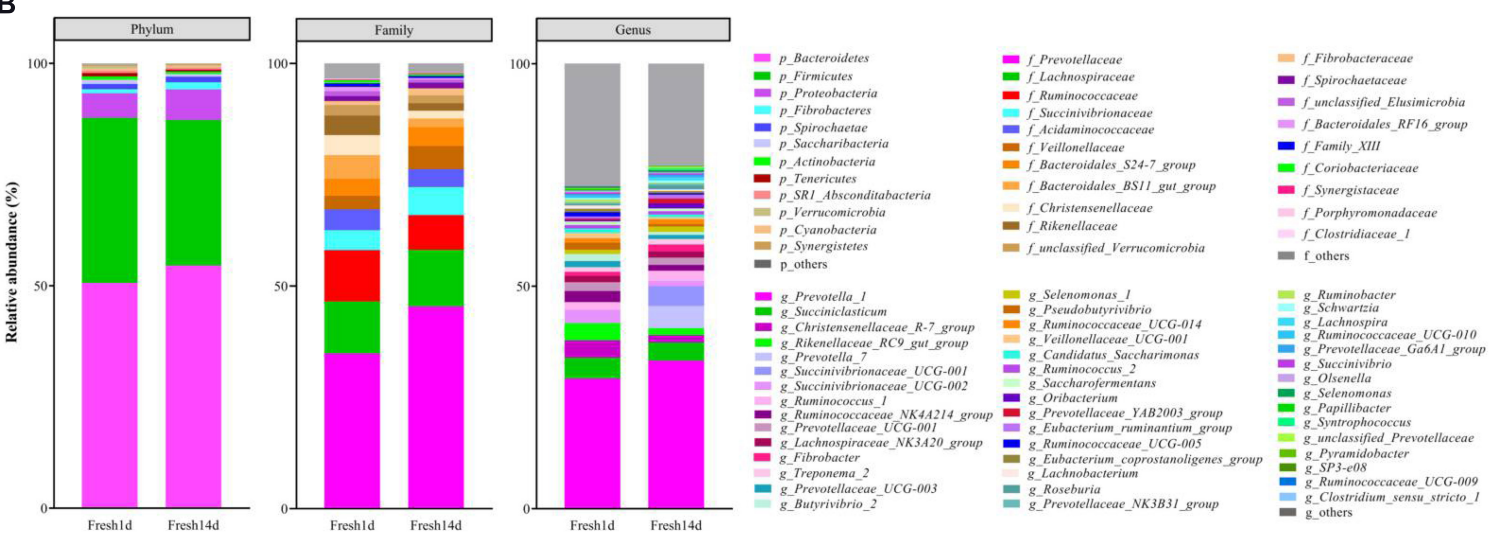

FIGURE 1 | The ruminal bacterial composition of fresh cows. (A) Relative distribution of the most dominant bacterial phyla, family and genera (relative abundance $>0.1 \%$ for all samples) for fresh cow samples. The pie chart inner ring represents the genus level, the middle ring represents the family level and the outer ring represents the phylum level. Different shades of color represent different bacteria. Numbers in brackets denote the average relative abundance of the bacteria across 40 samples. (B) Stacked bar graphs of the average relative abundances of phyla, family and genus (relative abundance $>0.1 \%$ at least one sample) for fresh cow on $\mathrm{d} 1$ and $\mathrm{d} 14$ after calving.

A

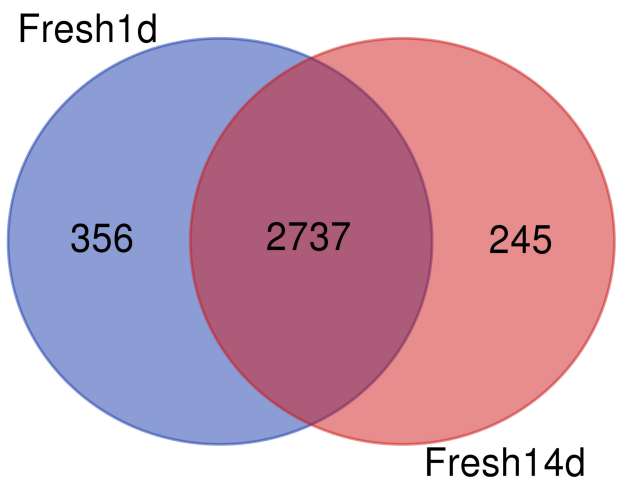

B LFI

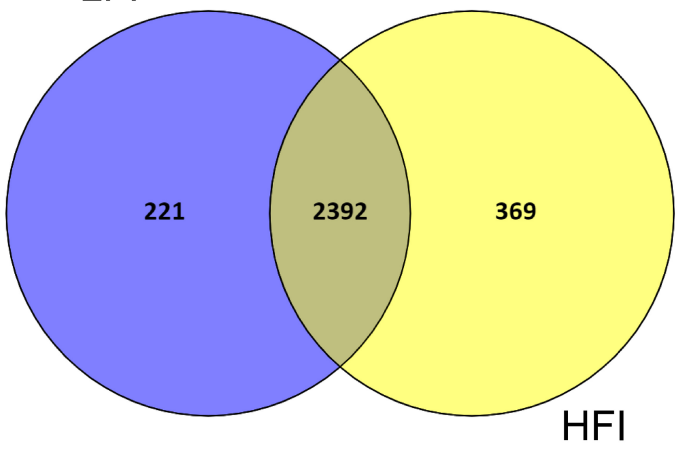

FIGURE 2 | (A) Venn diagram plot of fresh cow samples. The core community for all fresh cow is defined as those OTUs present in all fresh animals cows for all sampling time. (B) Venn diagram plot of LFI and HFI cow samples. The core community for LFI and HFI cows is defined as those OTUs present in LFI and HFI cow at $14 \mathrm{~d}$ after calving.

as showed in Figure 4. We found a total of 24 OTUs that were significantly $(P<0.05)$ correlated with DMI; of them, 8 OTUs negatively correlated with DMI, 4 of which were in the genus Prevotella_1 $(P<0.05)$ and 3 of which were in the family Bacteroidales_S24-7_group $(P<0.05)$. There were 15 OTUs positively correlated with DMI, of which 3 were 


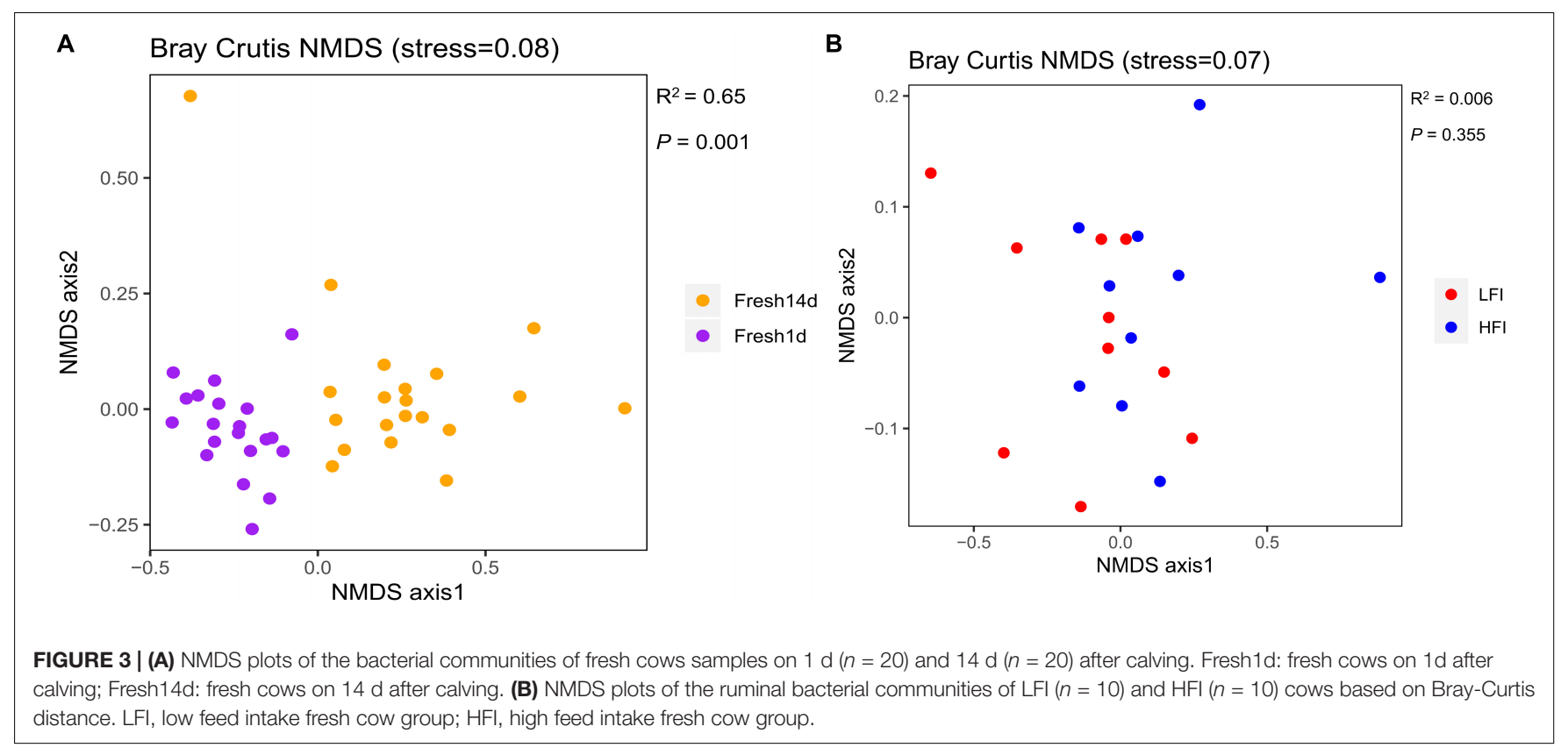

TABLE 3 | Significantly different phyla and families (relative abundance > 0.1\%) within the rumen microbiota by lactation period and DMI as determined by the Wilcoxon test.

\begin{tabular}{|c|c|c|c|c|c|c|c|c|}
\hline \multirow[t]{2}{*}{ Phylum/Family } & \multicolumn{2}{|c|}{ Lactation } & \multirow[t]{2}{*}{ SEM } & \multirow[t]{2}{*}{$P$-value } & \multicolumn{2}{|c|}{ DMI } & \multirow[t]{2}{*}{ SEM } & \multirow[t]{2}{*}{$P$-value } \\
\hline & Fresh1 & Fresh14d & & & LFI & HFI & & \\
\hline Actinobacteria & 0.74 & 0.51 & 0.06 & 0.040 & 0.39 & 0.63 & 0.07 & 0.089 \\
\hline Coriobacteriaceae & 0.66 & 0.43 & 0.05 & 0.011 & 0.32 & 0.54 & 0.06 & 0.045 \\
\hline Bacteroidetes & 50.65 & 54.56 & 1.17 & 0.210 & 52.04 & 57.07 & 1.68 & 0.143 \\
\hline Bacteroidales_BS11_gut_group & 5.30 & 1.95 & 0.42 & $<0.001$ & 1.57 & 2.34 & 0.27 & 0.280 \\
\hline Bacteroidales_Incertae_Sedis & 0.55 & 0.27 & 0.03 & 0.002 & 0.23 & 0.30 & 0.03 & 0.315 \\
\hline Bacteroidales_RF16_group & 1.00 & 0.40 & 0.08 & 0.006 & 0.33 & 0.47 & 0.09 & 0.406 \\
\hline Bacteroidales_UCG-001 & 0.17 & 0.08 & 0.02 & 0.035 & 0.04 & 0.11 & 0.02 & 0.256 \\
\hline Marinilabiaceae & 0.12 & 4.18E-03 & 0.02 & 0.002 & 2.09E-03 & $6.28 \mathrm{E}-03$ & $1.49 \mathrm{E}-03$ & 0.087 \\
\hline Porphyromonadaceae & 0.21 & 0.02 & 0.04 & 0.002 & 7.53E-03 & 0.04 & 0.02 & 0.516 \\
\hline Prevotellaceae & 34.89 & 45.45 & 1.70 & 0.002 & 44.07 & 46.82 & 2.07 & 0.315 \\
\hline Rikenellaceae & 4.35 & 1.68 & 0.32 & $<0.001$ & 1.27 & 2.09 & 0.29 & 0.315 \\
\hline Firmicutes & 37.11 & 32.69 & 1.47 & 0.054 & 34.16 & 31.22 & 1.40 & 0.481 \\
\hline Christensenellaceae & 4.53 & 1.76 & 0.32 & 0.002 & 1.30 & 2.22 & 0.35 & 0.063 \\
\hline Clostridiales_vadinBB60_group & 0.11 & 0.03 & 0.01 & 0.010 & 0.02 & 0.04 & 8.91E-03 & 0.092 \\
\hline Family_XIII & 0.80 & 0.46 & 0.04 & 0.002 & 0.46 & 0.46 & 0.03 & 0.791 \\
\hline ODP1230B8.23 & 0.17 & 0.08 & 0.03 & 0.011 & 0.02 & 0.13 & 0.05 & 0.140 \\
\hline Ruminococcaceae & 11.49 & 7.77 & 0.55 & 0.007 & 7.96 & 7.58 & 0.53 & 0.796 \\
\hline Veillonellaceae & 3.03 & 5.16 & 0.34 & 0.003 & 5.46 & 4.86 & 0.45 & 0.529 \\
\hline Proteobacteria & 5.53 & 6.90 & 1.17 & 0.840 & 9.36 & 4.43 & 1.23 & 0.043 \\
\hline Desulfovibrionaceae & 0.34 & 0.14 & 0.03 & $<0.001$ & 0.13 & 0.15 & 0.02 & 0.940 \\
\hline Succinivibrionaceae & 4.49 & 6.30 & 0.73 & 0.610 & 8.89 & 3.71 & 1.25 & 0.043 \\
\hline Synergistetes & 0.18 & 0.19 & 0.08 & 0.005 & 0.10 & 0.28 & 0.09 & 0.186 \\
\hline Synergistaceae & 0.18 & 0.19 & 0.04 & 0.060 & 0.10 & 0.28 & 0.09 & 0.186 \\
\hline Tenericutes & 0.75 & 0.47 & 0.06 & 0.005 & 0.44 & 0.50 & 0.06 & 0.880 \\
\hline Anaeroplasmataceae & 0.39 & 0.24 & 0.03 & 0.049 & 0.19 & 0.28 & 0.04 & 0.344 \\
\hline
\end{tabular}

in the Treponema_2 $(P<0.05)$ and 2 were in the family Prevotellaceae $(P<0.05)$. There was one OTUs identified as belonging to the Defluviitaleaceae_UCG-011 that was negatively
$(P<0.05)$ associated with DMI. In addition, OTUs within the Prevotellaceae_UCG-001, Lachnobacterium, and Olsenella were significantly and positively $(P<0.05)$ correlated with DMI. 
TABLE 4 | Significantly different genera (relative abundance > 0.1\%) within the rumen microbiota by lactation period and DMI as determined by the Wilcoxon test.

\begin{tabular}{|c|c|c|c|c|c|c|c|c|}
\hline \multirow[t]{2}{*}{ Genera } & \multicolumn{2}{|c|}{ Lactation } & \multirow[t]{2}{*}{ SEM } & \multirow[t]{2}{*}{$P$-value } & \multicolumn{2}{|c|}{ DMI } & \multirow[t]{2}{*}{ SEM } & \multirow[t]{2}{*}{$P$-value } \\
\hline & Fresh1d & Fresh14d & & & LFI & HFI & & \\
\hline Phocaeicola & 0.55 & 0.27 & 0.03 & $<0.001$ & 0.23 & 0.30 & 0.03 & 0.315 \\
\hline Prevotella_7 & 0.06 & 5.00 & 1.02 & $<0.001$ & 7.80 & 2.19 & 1.90 & 0.344 \\
\hline Prevotellaceae_Ga6A1_group & 0.22 & 0.49 & 0.04 & 0.003 & 0.41 & 0.58 & 0.06 & 0.290 \\
\hline Prevotellaceae_UCG-003 & 1.37 & 0.91 & 0.09 & 0.017 & 0.70 & 1.13 & 0.12 & 0.112 \\
\hline Prevotellaceae_YAB2003_group & 0.32 & 1.07 & 0.10 & $<0.001$ & 1.13 & 1.00 & 0.15 & 0.912 \\
\hline Rikenellaceae_RC9_gut_group & 3.84 & 1.54 & 0.28 & $<0.001$ & 1.19 & 1.88 & 0.24 & 0.315 \\
\hline SP3-e08 & 0.20 & 0.06 & 0.03 & $<0.001$ & 0.01 & 0.11 & 0.05 & 0.494 \\
\hline Anaerotruncus & 0.14 & 0.04 & 0.01 & $<0.001$ & 0.05 & 0.03 & 0.01 & 1.000 \\
\hline Anaerovorax & 0.20 & 0.09 & 0.01 & $<0.001$ & 0.10 & 0.09 & 0.01 & 0.677 \\
\hline Butyrivibrio_2 & 1.57 & 0.66 & 0.10 & $<0.001$ & 0.65 & 0.68 & 0.06 & 0.971 \\
\hline Christensenellaceae_R-7_group & 3.90 & 1.66 & 0.28 & $<0.001$ & 1.20 & 2.11 & 0.34 & 0.070 \\
\hline Coprococcus_2 & 0.02 & 0.06 & 0.01 & 0.003 & 0.07 & 0.06 & 0.01 & 0.426 \\
\hline Erysipelotrichaceae_UCG-002 & 2.09E-04 & 0.08 & 0.02 & 0.001 & 0.14 & 0.01 & 0.03 & 0.038 \\
\hline Eubacterium_coprostanoligenes_group & 0.79 & 0.45 & 0.05 & 0.003 & 0.42 & 0.48 & 0.03 & 0.406 \\
\hline Eubacterium_hallii_group & 0.16 & 0.12 & 0.01 & 0.018 & 0.09 & 0.14 & 0.02 & 0.173 \\
\hline Eubacterium_ruminantium_group & 0.47 & 0.91 & 0.07 & 0.008 & 1.01 & 0.81 & 0.11 & 0.529 \\
\hline Eubacterium_uniforme & $6.28 \mathrm{E}-04$ & 0.10 & 0.02 & $<0.001$ & 0.13 & 0.08 & 0.03 & 0.344 \\
\hline Lachnoclostridium_1 & 0.11 & 0.17 & 0.01 & 0.006 & 0.18 & 0.15 & 0.01 & 0.344 \\
\hline Lachnoclostridium_12 & 0.03 & 0.09 & 0.01 & 0.017 & 0.10 & 0.07 & 0.02 & 0.384 \\
\hline Lachnospira & 0.05 & 0.67 & 0.09 & $<0.001$ & 0.92 & 0.41 & 0.16 & 0.075 \\
\hline Lachnospiraceae_ND3007_group & 0.39 & 0.18 & 0.04 & 0.001 & 0.21 & 0.16 & 0.05 & 0.850 \\
\hline Lachnospiraceae_NK4A136_group & 0.13 & 0.07 & 0.01 & 0.013 & 0.08 & 0.06 & 0.01 & 0.449 \\
\hline Lachnospiraceae_XPB1014_group & 0.47 & 0.14 & 0.03 & $<0.001$ & 0.10 & 0.17 & 0.03 & 0.256 \\
\hline Lactobacillus & 0.01 & 0.07 & 0.01 & 0.002 & 0.10 & 0.04 & 0.03 & 0.677 \\
\hline Megasphaera & 0.01 & 0.11 & 0.02 & $<0.001$ & 0.14 & 0.08 & 0.03 & 0.363 \\
\hline Moryella & 0.31 & 0.22 & 0.02 & 0.010 & 0.22 & 0.23 & 0.02 & 0.970 \\
\hline Oribacterium & 0.32 & 1.13 & 0.14 & $<0.001$ & 1.42 & 0.84 & 0.25 & 0.529 \\
\hline Papillibacter & 0.42 & 0.06 & 0.05 & $<0.001$ & 0.05 & 0.07 & 0.01 & 0.384 \\
\hline probable_genus_10 & 0.18 & 0.11 & 0.01 & 0.039 & 0.12 & 0.11 & 0.02 & 0.850 \\
\hline Pseudobutyrivibrio & 1.59 & 0.53 & 0.14 & $<0.001$ & 0.46 & 0.59 & 0.06 & 0.353 \\
\hline Ruminococcaceae_NK4A214_group & 2.51 & 1.30 & 0.14 & $<0.001$ & 1.21 & 1.39 & 0.12 & 0.481 \\
\hline Ruminococcaceae_UCG-002 & 0.21 & 0.11 & 0.02 & 0.013 & 0.11 & 0.12 & 0.02 & 0.496 \\
\hline Ruminococcaceae_UCG-005 & 0.95 & 0.31 & 0.10 & 0.002 & 0.30 & 0.32 & 0.07 & 0.393 \\
\hline Ruminococcaceae_UCG-010 & 0.59 & 0.13 & 0.06 & $<0.001$ & 0.11 & 0.16 & 0.03 & 0.054 \\
\hline Schwartzia & 0.32 & 0.52 & 0.04 & 0.004 & 0.53 & 0.51 & 0.04 & 0.623 \\
\hline Selenomonas & 4.81E-03 & 0.50 & 0.07 & $<0.001$ & 0.65 & 0.35 & 0.13 & 0.315 \\
\hline Selenomonas_3 & 0.00 & 0.10 & 0.02 & $<0.001$ & 0.15 & 0.07 & 0.03 & 0.103 \\
\hline Veillonellaceae_UCG-001 & 1.24 & 0.47 & 0.09 & $<0.001$ & 0.44 & 0.50 & 0.05 & 0.473 \\
\hline Desulfovibrio & 0.33 & 0.14 & 0.03 & $<0.001$ & 0.13 & 0.15 & 0.02 & 0.910 \\
\hline Ruminobacter & 0.68 & 0.17 & 0.09 & $<0.001$ & 0.18 & 0.15 & 0.06 & 0.520 \\
\hline Succinivibrionaceae_UCG-001 & 1.05E-03 & 4.45 & 0.71 & $<0.001$ & 6.48 & 2.42 & 1.24 & 0.162 \\
\hline Succinivibrionaceae_UCG-002 & 2.92 & 1.16 & 0.36 & 0.005 & 1.59 & 0.72 & 0.40 & 0.472 \\
\hline Candidatus_Saccharimonas & 0.98 & 0.60 & 0.06 & 0.005 & 0.57 & 0.63 & 0.05 & 0.529 \\
\hline Pyramidobacter & 0.13 & 0.15 & 0.03 & 0.018 & 0.09 & 0.21 & 0.06 & 0.186 \\
\hline Anaeroplasma & 0.39 & 0.24 & 0.03 & 0.036 & 0.19 & 0.28 & 0.04 & 0.344 \\
\hline
\end{tabular}

We also found 11 OTUs that were significantly correlated with milk yield; of them, 6 OTUs were significantly and negatively $(P<0.05)$ associated with milk yield, and belonged to the genera Lachnospiraceae_NK3A20_group, Coprococcus_1, Oribacterium, Mogibacterium and family
Bacteroidales_BS11_gut_group and Prevotellaceae. There were 5 OTUs significantly and positively $(P<0.05)$ correlated with milk yield and belonged to the genera Prevotella_1, Anaeroplasma, Treponema_2, and Eubacterium_coprostanoligenes_group. Additionally, we identified a significant and negative correlation 
between DMI/milk yield and the relative abundance of OTUs within Prevotella_1 and the family Bacteroidales_S24-7_group $(P<0.05)$.

For VFAs, we found that acetate concentration was postively correlated with the relative abundance of OTU2072 (Anaeroplasma, $r=0.578, \quad P=0.007$ ) and OTU587 (Treponema_2, $r=0.550, P=0.012)$. We also found that propionate concentration was negatively correlated with the relative abundance of four OTUs, including OTU2019 (Butyrivibrio_2, $r=-0.654, P=0.003$ ), OTU742 (Prevotella_1, $r=-0.469, P=0.049$ ), OTU928 (Prevotella_1, $r=-0.523$, $P=0.026$ ), and OTU2499 (Christensenellaceae_R-7_group, $r=-0.476, P=0.046)$. The butyrate concentration was negatively and significantly $(P<0.05)$ correlated with OTUs within Butyrivibrio_2, Candidatus_Saccharimonas, and Prevotella_1. OTUs within Anaeroplasma and the family Bacteroidales_S247 group were significantly and positively $(P<0.05)$ correlated with valerate concentration, while OTUs within Acetitomaculum, Butyrivibrio_2, Candidatus_Saccharimonas, and Christensenellaceae_R-7_group were significantly and negatively $(P<0.05)$ correlated with valerate concentration. The isovalerate concentration was positively correlated with OTU1731 (Fibrobacter, $r=0.522, P=0.018$ ). The AP ratio was positively correlated with the relative abundances of OTU2019 (Butyrivibrio_2, $r=0.571, P=0.013$ ), OTU2297 (Christensenellaceae_R-7_group, $\mathrm{r}=0.514, P=0.029$ ), OTU2499 (Christensenellaceae_R-7_group, $r=0.491, P=0.038$ ), OTU2457 (Mogibacterium, $r=0.502, P=0.034$ ) and OTU928 (Prevotella_1, $r=0.504, P=0.033)$.

\section{DISCUSSION}

The objective of this study was to characterize the ruminal microbiota during the freshening period and to determine the impact of DMI in shaping its dynamics. We designed this experiment to follow the rumen microbiota within the first 14 days after calving in a group of fresh cows and to compare low and high DMI cows from this group. Given that low DMI in fresh cows is known to result in reduced lactation efficiency and increased risk for host metabolic syndromes, understanding the dynamics of the ruminal microbiota during this period may provide a framework for managing this critical transition period.

Consistent with the known changes in both host metabolism and the endocrine system across gestation and lactation, it is perhaps not surprising that we observed significant differences in the ruminal bacterial community and rumen fermentation index in fresh cows between days 1 and 14. This is also likely due to the significant differences in the diet fed to transition animals, which differs substantially from the beginning to the end of this period. Recently it was shown that lactation has a far greater impact in shaping the ruminal microbiota in dairy cows than host genetics (Bainbridge et al., 2016). Additionally, Pitta et al. (2014) found that the ruminal microbiota of lactation cows 1-3 days after calving was most similar to the ruminal microbiota of prepartum cows. Thus, the shifts in the ruminal bacterial community of fresh cows from 1 to 14 days observed here is likely due to the interaction of lactation and diet.

Compared to Fresh1d cows, the Fresh14d cows harbored a higher relative abundance of Prevotellaceae, Veillonellaceae, and bacteria within these families are known to degrade and ferment carbohydrates into VFAs (Zhang et al., 2018). We also found significantly higher relative abundances of Succinivibrionaceae_UCG-001, which was increased more than 4,000-fold in Fresh14d cows. This is in agreement with observations indicating that members of this genera utilize hydrogen to produce succinate, which can be converted to propionate (McCabe et al., 2015). This purported in propionate may contribute to the observed decrease in AP. Moreover, we found higher levels of acetate, propionate, valerate, total VFAs, and a lower AP ratio in the rumen from Fresh14d cows, relative to Fresh1d cows, which likely reflects a stronger fermentation capacity of the Fresh14d ruminal microbiota.

In addition to our findings on the temporal dynamics of the rumen microbiota in fresh cows, our study also considered the impact of DMI on the rumen microbiota of fresh cows. Here, we found that increased DMI was associated with lower relative abundances of Erysipelotrichaceae_UCG-002, within the family Erysipelotrichaceae, and higher relative abundances of Ruminococcaceae_UCG-010, within the family Ruminococcaceae. These findings are in accordance with a previous study of low, medium, and high feed intake cows during early lactation which found decreased numbers of Erysipelotrichaceae and increased numbers of Ruminococcaceae in high feed intake cows (Li et al., 2020). More recently, a study on the feed efficiency of dairy cows found that increased milk production was associated with higher relative abundances of bacteria in the Bacteroidales, Lachnospiraceae, Ruminococcaceae, and Prevotella (Shabat et al., 2016).

Given the effect of DMI on the rumen bacterial community, it is not surprising that specific bacterial species are strongly correlated with DMI. Here, we observed a strong correlation between DMI and bacteria in the families Prevotellaceae, Ruminnococcaceae, unclassified_Bacteroidales_S11_gut_group, and Lachnospiraceae. This is in accordance with other studies that also found a strong correlation between DMI and bacteria in the families Prevotellaceae and Ruminnococcaceae (Jami et al., 2014). Other work demonstrating the heritability of OTUs within the Succinivibrionaceae, Megasphaera, Selenonmonas, Oscillospira, and unclassified BS11, as it relates to DMI in beef cattle (Li et al., 2019), also support our findings. Related to this, our study also found that OTUs associated with high DMI fresh cows were the core microbiota in LFI and HFI cows. We note that many of these are consistent with previously reported OTUs found to be heritable in high DMI beef cattle, including bacteria in the Prevotella and Lanchnospirraceae (Sasson et al., 2017). In our study, we also found a strong and positive correlation between milk yield and OTUs within Prevotella_1, Anaeroplasm and Treponema_2. This is in accordance with other studies which also found that Prevotella, unclassified_Bacteroidales_S24-7 and Succinivibrionaceae were strongly and positively correlated with milk yield in lactating dairy cows (Indugu et al., 2017). Given 


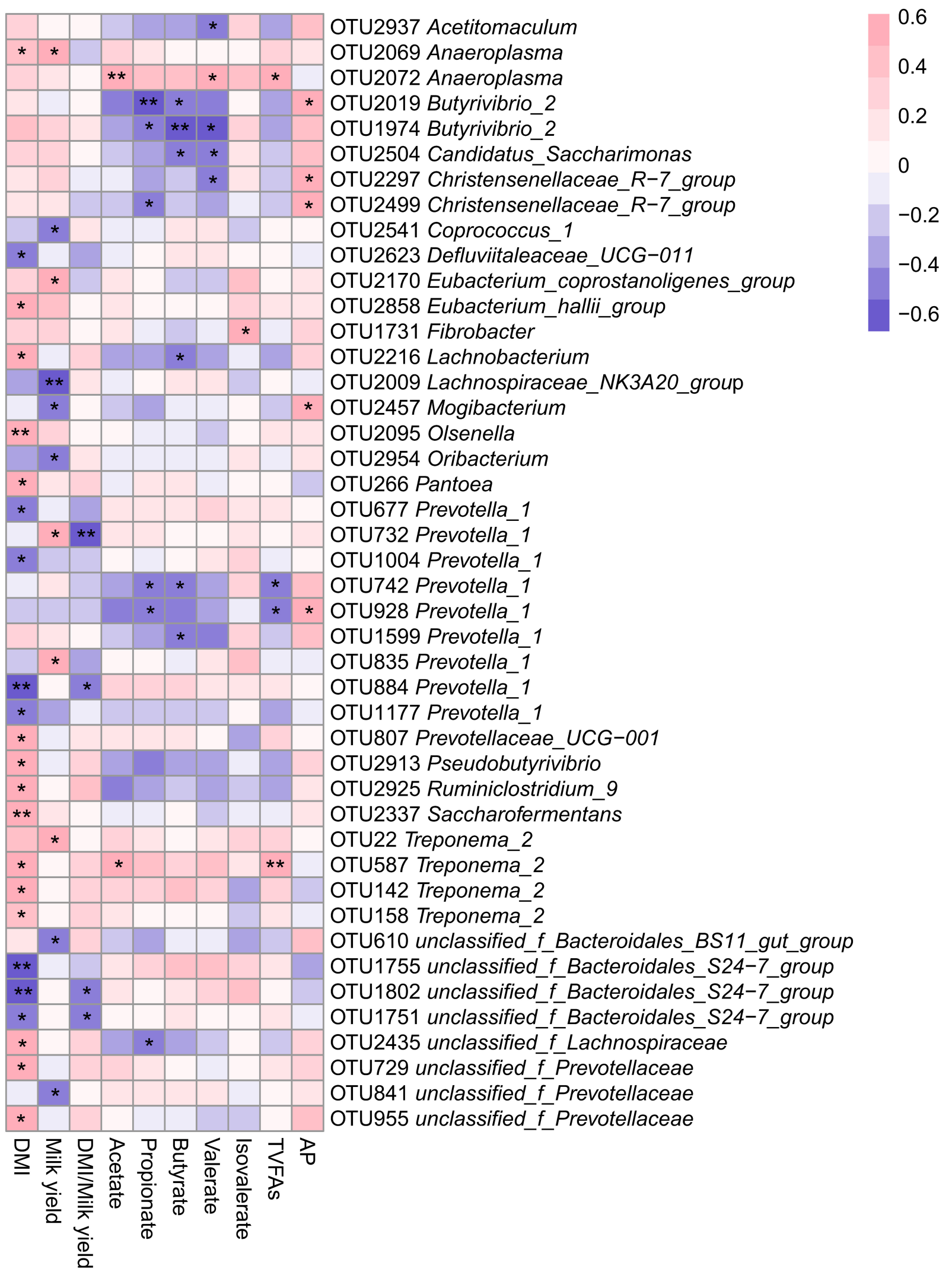

FIGURE 4 | Heatmap of OTUs (relative abundance $>0.01 \%$ in at all samples) significantly associated with production and rumen fermentative parameters in LFI and HFI cows, as determined by Spearman's correlation analysis. ${ }^{*} 0.01<P<0.05,{ }^{* *} 0.001<P<0.01$ 
these findings future work should further investigate these bacteria as potential targets for improving DMI in fresh cows.

Considering the essential role of the rumen bacteria in fermenting plant material into VFAs (Kittelmann et al., 2013), which have a direct effect on milk production (Hurtaud et al., 1995; Brulc et al., 2009), documenting the rumen microbiota in early lactation may help in better understanding the impact of the rumen microbiota on production traits. Moreover, the rumen microbiota during the freshening period may serve as a predictor of future production and may allow for manipulation in order to improve long term milk production. The results presented here have identified a number of specific bacterial taxa associated with both low and high DMI in fresh cows over time, and many of these may serve as potential targets for mitigating the challenges associated with low DMI cows during the freshening period. However, future work using more functional approaches, such as metagenomics and metatranscriptomics, should be conducted to better understand the interaction between rumen microbiome and DMI in fresh cows.

\section{CONCLUSION}

In summary, the results of this study provide novel evidence for an alteration of the microbiome in the rumen of fresh cows from 1 to 14 days after calving. We found that the ruminal microbiota and its associated fermentation patterns differed during this period and that the relative abundance of many VFA-producing microbes within the Prevotellaceae, Lactobacillaceae, and Veillonellaceae were dramatically increased in Fresh14d cows compared with Fresh1d cows. These findings indicate a potential stronger ability to ferment dietary substrates by the rumen microbiota of Fresh14d cows than that of Fresh1d cows. Additionally, we found limited differences between the ruminal microbiota of LFI and HFI groups, thereby reflecting the limited role of DMI on shaping the rumen microbiota during the freshening period. Furthermore, a strong relationship between the relative abundances of specific OTUs and host production traits suggests the possibility to predict downstream host production using the rumen microbiota. This could lead to approaches for manipulating the rumen microbiota to improve DMI and milk production in dairy cows during the transition period. Future studies should investigate the relationship between the rumen microbiota and DMI across different environments in an integrative manner that incorporates both host genetics and functional metagenomics in the rumen.

\section{REFERENCES}

Bainbridge, M. L., Cersosimo, L. M., Wright, A. D., and Kraft, J. (2016). Rumen bacterial communities shift across a lactation in Holstein, Jersey and Holstein $\mathrm{x}$ Jersey dairy cows and correlate to rumen function, bacterial fatty acid composition and production parameters. FEMS Microbiol. Ecol. 92:fiw059. doi: 10.1093/femsec/fiw059

\section{DATA AVAILABILITY STATEMENT}

The datasets presented in this study can be found in online repositories. The names of the repository/repositories and accession number(s) can be found below: https://www.ncbi.nlm. nih.gov/, PRJNA599409.

\section{ETHICS STATEMENT}

The animal experiments and study protocols described in this study were approved by the Institutional Animal Care and Use Committee of the College of Animal Science and Technology (Project number 31772628) at China Agricultural University, Beijing, China.

\section{AUTHOR CONTRIBUTIONS}

SJ, SL, and SH conceived and designed the study. SH and FW collected all samples used in this study. SH performed the data analysis and wrote the manuscript with contributions from GS. All authors read and approved the final manuscript.

\section{FUNDING}

This research was supported by the Key Research and Development Project of Ningxia Hui Autonomous Region grant numbers 2018BBF33006 and 2018YFD0501600 from the National Key Research and Development Program of China. GS was supported by a US Department of Agriculture National Institute of Food and Agriculture Hatch project WIS02007.

\section{ACKNOWLEDGMENTS}

We would like to thank the staff at the Zhongdi Breeding Stock Co., Ltd., for daily animal care. We also thank all members of the Li laboratory for their support and help in collecting samples. Finally, we thank Jie Huang from Yangzhou Univerisity, Yangzhou, China, for collecting samples and feeding animals.

\section{SUPPLEMENTARY MATERIAL}

The Supplementary Material for this article can be found online at: https://www.frontiersin.org/articles/10.3389/fmicb. 2021.630605/full\#supplementary-material

Benjamini, Y., and Hochberg, Y. (1995). Controlling the false discovery rate: a practical and powerful approach to multiple testing. J. R. Statis. Soc. 57, 289-300. doi: 10.1111/j.2517-6161.1995.tb02031.x

Brulc, J. M., Antonopoulos, D. A., Miller, M. E., Wilson, M. K., Yannarell, A. C., Dinsdale, E. A., et al. (2009). Gene-centric metagenomics of the fiber-adherent bovine rumen microbiome reveals forage specific glycoside hydrolases. Proc. Nalt. Acad. Sci. U.S.A. 106, 1948-1953. doi: 10.1073/pnas.0806191105 
Caporaso, J. G., Kuczynski, J., Stombaugh, J., Bittinger, K., Bushman, F. D., Costello, E. K., et al. (2010). QIIME allows analysis of highthroughput community sequencing data. Nat. Methods 7, 335-336. doi: 10.1038/ nmeth0510-335

Caporaso, J. G., Lauber, C. L., Walters, W. A., Berg-Lyons, D., Huntley, J., Fierer, N., et al. (2012). Ultra-high-throughput microbial community analysis on the Illumina HiSeq and MiSeq platforms. ISME J. 6, 1621-1624. doi: 10.1038/ismej. 2012.8

Cole, J. R., Wang, Q., Cardenas, E., Fish, J., Chai, B., Farris, R. J., et al. (2009). The Ribosomal database project: improved alignments and new tools for rRNA analysis. Nucleic Acids Res. 37, D141-D145. doi: 10.1093/nar/gkn879

Edgar, R. C. (2010). Search and clustering orders of magnitude faster than BLAST. Bioinformatics 26, 2460-2461. doi: 10.1093/bioinformatics/btq461

Edgar, R. C., Haas, B. J., Clemente, J. C., Quince, C., and Knight, R. (2011). UCHIME improves sensitivity and speed of chimera detection. Bioinformatics 27, 2194-2200. doi: 10.1093/bioinformatics/btr381

Erwin, E., Marco, G., and Emery, E. (1961). Volatile fatty acid analyses of blood and rumen. J. Dairy Sci. 44, 1768-1771.

Ferguson, J. D. (2001). Milk Urea Nitrogen. 2681-2692. Available online at: http: //cahpwww.vet.upenn.edu/doku.php/dairycattle:mun. (accessed July 28, 2014).

Flint, H. J., Bayer, E. A., Rincon, M. T., Lamed, R., and White, B. A. (2008). Polysaccharide utilization by gut bacteria: potential for new insights from genomic analysis. Nat. Rev. Microbiol. 6, 121-131. doi: 10.1038/nrmicro1817

Hurtaud, C., Rulquin, H., and Verite, R. (1995). Effect of infused volatile fatty acids and caseinate on milk composition and coagulation in dairy cows. J. Dairy Sci. 76, 3011-3020. doi: 10.3168/jds.S0022-0302(93)77640-7

Indugu, N., Vecchiarelli, B., Baker, L. D., Ferguson, J. D., Vanamala, J. K. P., and Pitta, D. W. (2017). Comparison of rumen bacterial communities in dairy herds of different production. BMC Microbiol. 17:190. doi: 10.1186/s12866-0171098-z

Jami, E., and Mizrahi, I. (2012). Composition and similarity of bovine rumen microbiota across individual animals. PLoS One 7:e33306. doi: 10.1371/journal. pone.0033306.g001

Jami, E., White, B. A., and Mizrahi, I. (2014). Potential role of the bovine rumen microbiome in modulating milk composition and feed efficiency. PLoS One 9:e85423. doi: 10.1371/journal.pone.0085423

Kittelmann, S., Seedorf, H., Walters, W. A., Clemente, J. C., Knight, R., Gordon, J. I., et al. (2013). Simultaneous amplicon sequencing to explore cooccurrence patterns of bacterial, archaeal and eukaryotic microorganisms in rumen microbial communities. PLoS One 8:e47879. doi: 10.1371/journal.pone. 0047879

Li, F., Li, C., Chen, Y., Liu, J., Zhang, C., Irving, B., et al. (2019). Host genetics influence the rumen microbiota and heritable rumen microbial features associate with feed efficiency in cattle. Microbiome 7:92. doi: 10.1186/s40168019-0699-1

Li, Y. Q., Xi, Y. M., Wang, Z. D., Zeng, H. F., and Han, Z. (2020). Combined signature of rumen microbiome and metabolome in dairy cows with different feed intake levels. J. Anim. Sci. 98, 1-15. doi: 10.1093/jas/skaa070

Lima, F. S., Oikonomou, G., Lima, S. F., Bicalho, M. L., Ganda, E. K., Filho, J. C., et al. (2015). Prepartum and postpartum rumen fluid microbiomes: characterization and correlation with production traits in dairy cows. Appl. Environ. Microbiol. 81, 1327-1337. doi: 10.1128/AEM.03138-14

Magoc, T., and Salzberg, S. L. (2011). FLASH: fast length adjustment of short reads to improve genome assemblies. Bioinformatics 27, 2957-2963. doi: 10.1093/ bioinformatics/btr507

McCabe, M. S., Cormican, P., Keogh, K., O’Connor, A., O’Hara, E., Palladino, R. A., et al. (2015). Illumina MiSeq phylogenetic amplicon sequencing shows a large reduction of an uncharacterised Succinivibrionaceae and an Increase of the Methanobrevibacter gottschalkii Clade in feed restricted cattle. PLoS One 10:e0133234. doi: 10.1371/journal.pone.0133234

Oksanen, J., Blanchet, F. G., Kindt, R., Legendre, P., Minchin, P. R., O’Hara, R. B., et al. (2015). Vegan: Community Ecology Package. R Package Version 2.2-1.
Pitta, D. W., Kumar, S., Vecchiarelli, B., Shirley, D. J., Bittinger, K., and Baker, L. D. (2014). Temporal dynamics in the ruminal microbiome of dairy cows during the transition period. J. Anim. Sci. 92, 4014-4022. doi: 10.2527/jas20147621

Quast, C., Pruesse, E., Yilmaz, P., Gerken, J., Schweer, T., Yarza, P., et al. (2013). The SILVA ribosomal RNA gene database project: improved data processing and web-based tools. Nucleic Acids Res. 41, D590-D596. doi: 10.1093/nar/gks1219

Ren, W., Wang, P., Yan, J., Liu, G., Zeng, B., Hussain, T., et al. (2017). Melatonin alleviates weanling stress in mice: involvement of intestinal microbiota. J. Pineal. Res. 64:e12448. doi: 10.1111/jpi.12448

Reynolds, C. K., Huntington, G. B., Tyrrell, H. F., and Reynolds, P. J. (1988). Net Metabolism of volatile fatty acids, $d-\beta$-hydroxybutyrate, nonesterified fatty acids, and blood gasses by portal-drained viscera and liver of lactating holstein cows. J. Dairy Sci. 71, 2395-2405. doi: 10.3168/jds.s0022-0302(88)79824-0

Roche, J. R., Bell, A. W., Overton, T. R., and Loor, J. J. (2013). Nutritional management of the transition cow in the 21st century-a paradigm shift in thinking. Anim. Prod. Sci. 53, 1000-1023. doi: 10.1071/an1 2293

Sasson, G., Kruger Ben-Shabat, S., Seroussi, E., Doron-Faigenboim, A., Shterzer, N., Yaacoby, S., et al. (2017). Heritable bovine rumen bacteria are phylogenetically related and correlated with the cow's capacity to harvest energy from its feed. mBio 8, e703-e717. doi: 10.1128/mBio.00703-17

Shabat, S. K., Sasson, G., Doron-Faigenboim, A., Durman, T., Yaacoby, S., Berg Miller, M. E., et al. (2016). Specific microbiome-dependent mechanisms underlie the energy harvest efficiency of ruminants. ISME J. 10, 2958-2972. doi: 10.1038 /ismej.2016.62

Shi, F. Y., Guo, N., Degen, A. A., Niu, J. H., Wei, H. Y., Jing, X. P., et al. (2020). Effects of level of feed intake and season on digestibility of dietary components, efficiency of microbial protein synthesis, rumen fermentation and ruminal microbiota in yaks. Anim. Feed Sci. Technol. 259:114359. doi: 10.1016/ j.anifeedsci.2019.114359

Weimer, P. J. (2015). Redundancy, resilience, and host specificity of the ruminal microbiota: implications for engineering improved ruminal fermentations. Front. Microbiol. 6:296. doi: 10.3389/fmicb.2015.00296

Wickham, H. (2009). ggplot2: Elegant Graphics for Data Analysis. New York, NY: Springer-Verlag.

Zhang, J. J., Kobert, K., Flouri, T., and Stamatakis, A. (2014). PEAR: a fast and accurate Illumina Paired-End reAd mergeR. Bioinformatics 30, 614-620. doi: 10.1093/bioinformatics/btt593

Zhang, L., Wu, W., Lee, Y. K., Xie, J., and Zhang, H. (2018). Spatial heterogeneity and co-occurrence of mucosal and luminal microbiome across swine intestinal tract. Front. Microbiol. 9:48. doi: 10.3389/fmicb.2018.0 0048

Zhu, Z., Kristensen, L., Difford, G. F., Poulsen, M., Noel, S. J., Abu Al-Soud, W., et al. (2018). Changes in rumen bacterial and archaeal communities over the transition period in primiparous Holstein dairy cows. J. Dairy Sci. 101, 9847-9862. doi: 10.3168/jds.2017-14366

Zhu, Z., Noel, S. J., Difford, G. F., Al-Soud, W. A., Brejnrod, A., Sorensen, S. J., et al. (2017). Community structure of the metabolically active rumen bacterial and archaeal communities of dairy cows over the transition period. PLoS One 12:e0187858. doi: 10.1371/journal.pone.0187858

Conflict of Interest: The authors declare that the research was conducted in the absence of any commercial or financial relationships that could be construed as a potential conflict of interest.

Copyright (C) 2021 Huang, Ji, Suen, Wang and Li. This is an open-access article distributed under the terms of the Creative Commons Attribution License (CC BY). The use, distribution or reproduction in other forums is permitted, provided the original author(s) and the copyright owner(s) are credited and that the original publication in this journal is cited, in accordance with accepted academic practice. No use, distribution or reproduction is permitted which does not comply with these terms. 